\title{
Hindrance to Day Care Laparoscopic Cholecystectomy in India
}

\author{
Bhaviya Bhargavan Nair Sarala ${ }^{1}$, Abhimanyu Kar ${ }^{2}$, Supriyo Ghatak ${ }^{3}$, Sumit Gulati ${ }^{4}$, Vishnu K Bhartia ${ }^{5}$, Pradeep K Nemani ${ }^{6}$
}

\begin{abstract}
Background: Laparoscopic cholecystectomy is considered "gold standard" for the treatment of gallstone disease. In spite of the increasing number of laparoscopic cholecystectomies being performed as day care surgery in the West, the surgeons of developing countries are reluctant to adopt this trend probably due to the inadequate resources and infrastructure which they consider a hindrance for safe discharge. Our study aims to assess the feasibility of day care laparoscopic cholecystectomies.

Materials and methods: This is a prospective observational study. All patients undergoing laparoscopic cholecystectomy were assessed postoperatively for dischargeability using post-anesthetic discharge scoring system (PADSS). We assessed the factors delaying the early discharge of laparoscopic cholecystectomy patients in terms of patient factors, intraoperative factors, postoperative factors, social factors, and logistic factors. Results: Of the total 88 patients, 57 (64.7\%) were dischargeable at 6 hours and 78 (88.6\%) were dischargeable at 24 hours. Factors found to affect dischargeability of patients at 6 hours were acute cholecystitis and increased duration of surgery. Difficulty of surgery and the use of drain had significant association with nondischargeability at 24 hours. Eighteen patients were fit for discharge by PADSS criteria but not discharged at 24 hours. Factors, which delayed the discharge of these patients, were continuation of intravenous antibiotics, delay in processing insurance, patients' unwillingness for early discharge, presence of drain, and surgeon's perceived fear of complications.

Conclusion: Sixty-five percent of all laparoscopic cholecystectomies can be performed as day care procedure safely. Patients with acute cholecystitis and patients requiring an operative time more than 104 minutes should be observed for 24 hours.

Keywords: Cholecystectomy, Day care surgery, Feasibility, Gallstone, Laparoscopic surgery, Safety.

World Journal of Laparoscopic Surgery (2020): 10.5005/jp-journals-10033-1396
\end{abstract}

\section{INTRODUCTION}

Laparoscopic cholecystectomy is considered the "gold standard" for the surgical treatment of gallstone disease. ${ }^{1}$ Earlier with open cholecystectomies, patients used to stay in the hospital for up to 6 days. $^{2}$ The introduction of laparoscopic cholecystectomy drastically shortened the hospital stay of cholecystectomy patients from 2-3 days. ${ }^{3}$ In the Western world, laparoscopic cholecystectomies are being performed as a day care surgery further reducing the stay of patients in hospital to less than a day.

Outpatient surgery, also known as ambulatory surgery, sameday surgery, day case, or day surgery, is surgery that does not require an overnight hospital stay. The potential for day care surgery has increased over the last few decades which can be attributed to the advances in surgical technologies and in the field of anesthesiology. Day care surgery allows a person to return home on the same day of the operation is performed and eliminates inpatient hospital admission, thereby reduces cost.

Day care laparoscopic cholecystectomy (DCLC) has already been widely accepted in Western countries and there are many studies on the safety and feasibility of DCLCs. However, these studies are from developed countries where there is advanced system for ambulatory surgeries. Because of differences in the quality of health care delivery, Western guidelines for day care surgery cannot be universally applied to developing countries.

In most part of eastern India, we still follow the traditional practice of discharging the laparoscopic cholecystectomy patients the next day or one day after the surgery probably due to lack of the literature regarding the safety of DCLCs from smaller centers in developing countries where the primary healthcare delivery and infrastructure is poor. For the effective implementation of DCLCs,
1,5,6 Department of General Surgery, Calcutta Medical Research Institute, Kolkata, West Bengal, India

${ }^{2-4}$ Department of Surgical Gastroenterology, Calcutta Medical Research Institute, Kolkata, West Bengal, India

Corresponding Author: Bhaviya Bhargavan Nair Sarala, Department of General Surgery, Calcutta Medical Research Institute, Kolkata, West Bengal, India, Phone: +4407404426473, e-mail: bavya_bs@yahoo.co.in How to cite this article: Sarala BBN, Kar A, Ghatak S, et al. Hindrance to Day Care Laparoscopic Cholecystectomy in India. World J Lap Surg 2020;13(1):16-20.

Source of support: Nil

Conflict of interest: None

first we require wide acceptance of overnight or 24 hour discharging units followed by gradual upgradation to day care centers. Further studies on the safety and efficacy of early discharge in laparoscopic cholecystectomies from smaller centers in India might give the surgeons the ever lacking confidence in DCLC.

In our institution, the traditional practice is to discharge the patients almost 48 hours after laparoscopic cholecystectomy. We wanted to find out whether it was safe and feasible to discharge these patients as day care (6 hours) or early ( 24 hours) in a center like ours where there is no dedicated unit for ambulatory surgery. In this prospective observational study, we aim to identify the patients suitable for DCLC, factors affecting the dischargeability at 6 and 24 hours, as well as to find out the factors delaying the discharge of LC patients more than 24 hours. As day care was not a traditional practice and we were apprehensive on the safety of discharging the LC patients as a day care, we did not discharge 
the patients as day care but continued to monitor all the patients till 24 hours to see whether any patients dischargeable at 6 hours developed any complication during their stay which would have led to a readmission if at all they were discharged as day care.

\section{Materials and Methods}

This is a prospective observational study conducted in the Department of General surgery at The Calcutta Medical Research Institute, Kolkata, over a period of 6 months. All consecutive patients, both male and female diagnosed with symptomatic gallstone disease undergoing laparoscopic cholecystectomy in the Department of General Surgery at The Calcutta Medical Research Institute, who gave consent for being a part of the study were included. Patients undergoing other combined procedures with laparoscopic cholecystectomy and pregnant patients undergoing laparoscopic cholecystectomy were excluded.

All patients who underwent laparoscopic cholecystectomy were evaluated preoperatively by a detailed history and the patient factors such as age, sex, BMI, comorbid conditions, past history of surgeries, and ASA score were noted.

Standard four-port laparoscopic cholecystectomy was performed in all the patients by the consultant surgeon. If the gallbladder were not taken out through the umbilical port, the patient was excluded from the study. Standard institutional protocol for postoperative analgesia was followed for all patients.

Several scoring systems have been developed for discharge after ambulatory anesthesia, one of the most widely used being the post-anesthesia discharge scoring system (PADSS), introduced by Chung and colleagues in 1995. ${ }^{4}$ We assessed patients at 6 hours and 24 hours postoperatively using PADSS. Maximal score was 10. Patients scoring 9 or 10 were fit for discharge (Table 1).

At 6 hours, the patients were assessed for their readiness for discharge. All the patients continued to be monitored till 24 hours, any complication during this time was noted, and they were again assessed at 24 hours for their readiness for discharge. If the patient met the criteria for discharge at 24 hours, he/she was discharged and if not discharged the reasons for delayed discharge were noted.

The statistical software SPSS version 20 has been used for the analysis. All categorical variables were analyzed using Fischer exact test and all continuous variables with Mann-Whitney test. An alpha level of $5 \%$ has been taken, i.e., if any $p$ value is less than 0.05 , it has been considered as significant. A multivariate logistic regression was performed including all variables with $p$ value $<0.05$ to predict the factors affecting discharge at 6 hours.

\section{Result and Analysis}

A total of 94 patients underwent laparoscopic cholecystectomy for symptomatic gallstone disease during the period of 6 months. Six patients were excluded from the study, one patient due to conversion to open cholecystectomy and five patients as the gallbladder was removed from the epigastric port due to technical difficulties. A total of 88 patients were included in the study.

Fifty-seven (64.7\%) patients were dischargeable at 6 hours. At 24 hours, 21 more patients were dischargeable making a total of 78 (88.6\%) patients dischargeable at 24 hours. Of the total 78 patients dischargeable at 24 hours, 18 (23.08\%) patients were not discharged due to various factors such as social factors, logistic factors, presence of drain, patient preference, and as surgeon anticipated complications. None of the patients who were dischargeable at
Table 1: Post-anesthesia discharge scoring system ${ }^{4}$

Vital signs: Vital signs must be stable and consistent with age and preoperative baseline

BP and pulse within 20\% of preoperative baseline $\quad 2$

BP and pulse $20-40 \%$ of preoperative baseline 1

BP and pulse $40 \%$ of preoperative baseline 0

Activity level: Patient must be able to ambulate at preoperative level Steady gait, no dizziness, or meets preoperative level 2

Requires assistance

1

Unable to ambulate

0

Nausea and vomiting: The patient should have minimal nausea and vomiting before discharge

Minimal: Successfully treated with PO medication $\quad 2$

Moderate: Successfully treated with IM medication $\quad 1$

Severe: Continues after repeated treatment

1

Pain: The patient should have minimal or no pain before discharge. The level of pain that the patient has should be acceptable to the patient. Pain should be controllable by oral analgesics. The location, type, and intensity of pain should be consistent with anticipated postoperative discomfort

Acceptability

Yes

2

No

1

Surgical bleeding: Postoperative bleeding should be consistent with expected blood loss for the procedure

Minimal: Does not require dressing change 2

Moderate: Up to two dressing changes required

Severe: More than three dressing changes required

1

Maximum score $=10$, score $\geq 9$ is fit for discharge

6 hours developed any complication during their period of stay in the hospital and continued to remain dischargeable at 24 hours.

Of all the variables analyzed, age, acute cholecystitis, diabetes mellitus (DM), hypertension (HTN), The American Society of Anesthesiologists (ASA) physical status classification system score, difficulty, use of drain, and duration of surgery were found to have significant association with dischargeability of the patients at 6 hours (Table 2). After logistic regression, only acute cholecystitis and duration of surgery had a significant association. From the ROC curve in our study, operating times more than 104 minutes can be considered as a predictive factor for failure of DCLC.

Of all the factors accessed, difficulty of surgery and use of drain were found to have significant association with dischargeability of the patients at 24 hours (Table 3 ). As these factors were interdependent, we did not perform a regression analysis.

Of the total 88 patients analyzed, 78 (88.6\%) patients dischargeable at 24 hours of which 60 patients were discharged at 24 hours. Rest of the 18 patients who were dischargeable were not discharged because of various factors such as continuation of IV antibiotics (1), logistic insurance (6), patient factor (1), presence of drain (8), social factors such as patient living at a far distance (1), and surgeon anticipating complication (1) (Fig. 1).

\section{Discussion}

The aim of our study was to detect the dischargeability of the laparoscopic cholecystectomy patients at 6 hours and 24 hours and to determine the factors affecting discharge. As day care laparoscopic cholecystectomy is not a routine practice 
Hindrance to Day Care Laparoscopic Cholecystectomy in India

Table 2: Factors affecting the dischargeability of patients at 6 hours

\begin{tabular}{lllll}
\hline Factors & p value & $\begin{array}{l}\text { p value after logistic } \\
\text { regression }\end{array}$ & Odds ratio $(95 \% \mathrm{Cl})$ & $\begin{array}{l}\text { Significance using logistic } \\
\text { regression }\end{array}$ \\
\hline Age & 0.007 & 0.901 & $0.997(0.947-1.049)$ & Not significant \\
Acute cholecystitis & 0.004 & 0.059 & $0.074(0.005-1.103)$ & Significant \\
DM & 0.001 & 0.455 & $0.456(0.058-3.578)$ & Not significant \\
HTN & 0.005 & 0.317 & $0.387(0.060-2.485)$ & Not significant \\
Duration of surgery & 0.001 & 0.023 & $0.976(0.955-0.997)$ & Significant \\
ASA & 0.001 & 0.971 & $1.037(0.153-7.022)$ & Not significant \\
Difficulty & $<0.001$ & 0.140 & $0.442(0.149-1.306)$ & Not significant \\
Use of drain & $<0.001$ & 0.214 & $0.270(0.034-2.129)$ & Not significant \\
\hline
\end{tabular}

DM, diabetes mellitus; HTN, hypertension; ASA, The American Society of Anesthesiologists (ASA) physical status classification system

Table 3: Factors affecting the dischargeability of patients at 24 hours

\begin{tabular}{lll}
\hline Factors & $p$ value & Significance \\
\hline Difficulty & 0.008 & Significant \\
Use of drain & 0.005 & Significant \\
\hline
\end{tabular}

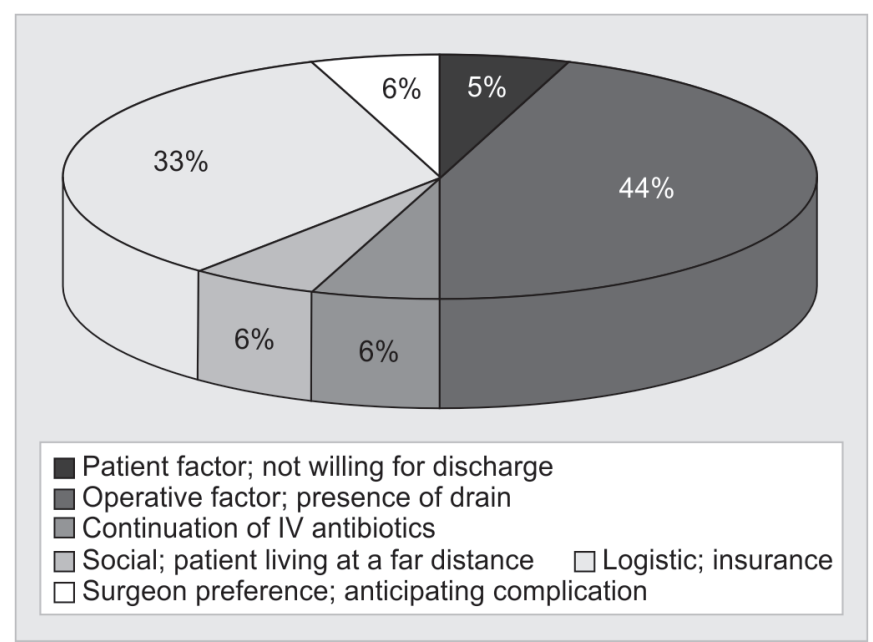

Fig. 1: Factors delaying discharge at 24 hours

at our institution, all the patients were assessed at 6 hours for dischargeability and then followed up till 24 hours till their discharge to see if they developed any complications which could have been a reason for readmission if at all these patients were discharged at 6 hours as day care. None of the patients who were dischargeable at 6 hours developed any complication during their period of stay in the hospital and continued to remain dischargeable at 24 hours.

Of the total 88 patients analyzed, 57 (64.7\%) patients were dischargeable at 6 hours and $78(88.6 \%)$ patients dischargeable at 24 hours. This means that $65 \%$ of all the laparoscopic cholecystectomies can be performed as DCLCs.

We found age ( $p$ value-0.007), acute cholecystitis (0.004), diabetes mellitus ( $p$ value -0.001$)$, hypertension ( $p$ value -0.005 ), ASA score ( $p$ value-0.001), duration of surgery (0.001), difficulty of surgery ( $p$ value $<0.001$ ), and use of drain ( $p$ value $<0.001$ ) to have significant association with dischargeability at 6 hours. After logistic regression analysis, only duration of surgery and acute cholecystitis were found to have a significant association (Table 2).

Previous studies stated age as a factor for failed discharge of DCLCs. ${ }^{5,6}$ In the study by Lledó et al. $(n=410)$, they found age of patient over 65 years $[p=0.021$; odds ratio $(\mathrm{OR})=2.225 ; 95 \%$ confidence interval $(\mathrm{Cl}), 1.130-4.381]$ as a predictive factor for overnight admission or failed discharge. ${ }^{5}$ In another study by Psaila et al., age over 50 years was one of the factors which adversely affected the same-day discharge. ${ }^{6}$ Contrary to these finding, a study on DCLCs in elderly showed that ambulatory LCs are safe in elderly patients ( $>65$ years). ${ }^{7}$ In our study, age was not a significant factor associated with dischargeability at 6 hours, after regression analysis. This is an indicator that even in elderly patients who have no other comorbidities, DCLCs might be safe.

In the study by Chauhan et al. although ASA grade III and IV were excluded, four of their day care cases required admission due to reasons like hypertension, COPD, and diabetes. ${ }^{8}$ They were of the opinion that these are dynamic diseases and likely to change between the interval between initial preanesthetic assessment and final surgery. In our study, although independently ASA score, diabetes, and hypertension had a significant association, and these variables did not show significant association after regression analysis. This might be due the interdependency of the variables such as age, hypertension, diabetes, and ASA score. A good initial preanesthetic evaluation and stringent patient selection for DCLCs should be followed to avoid potentially dangerous outcomes and decrease the number of cancellations of day care surgeries.

In our study, 53 (60.23\%) patients had easy surgery, 25 (28.41\%) patients had moderately difficult, and $10(11.36 \%)$ patients had very difficult surgery. Difficulty of surgery independently had a significant association with dischargeability 6 hours. The lack of the significant association after regression analysis may be due to its association with duration of surgery. Lledó et al. in their study had similar findings and identified "dissection difficulty" as one of the predictive factors related to overnight stay in DCLCs. ${ }^{9}$

Use of drain had a significant association with dischargeability at 6 hours in the initial analysis. This might be attributed to the increased pain experienced and the difficulty to ambulate in the patients with drain in situ. After regression analysis, the association was found to be not significant. This lack of significance after regression analysis might be due to the association between difficulty of surgery and usage of drain. We had used drain almost routinely for all the difficult cases. The nondischargeability in patients with drain might be mainly due to the difficulty of the surgery rather than the presence of drain.

The mean operative time in our study was 97.39 minutes (SD = 38.03). In our study, duration of surgery was a statistically significant independent factor associated with dischargeability at 6 hours. One of the studies had showed operation duration superior to 60 minutes to be a predictive factor to overnight admission. ${ }^{5}$ From 
the ROC curve in our study, operating times more than 104 minutes can be considered as a predictive factor for failure of DCLC.

Acute cholecystitis had a significant association with dischargeability at 6 hours with a $p$ value of 0.004 . Patients with acute cholecystitis might not be ideal candidate for day care surgeries and can be considered as exclusion criteria in selection of patients for DCLCs.

Of all the factors accessed, difficulty of surgery and use of drain were found to have significant association with dischargeability of the patients at 24 hours. Sherigar et al. in their study showed $3.4 \%$ readmission after discharge in the first phase due to wound related problems and surgical complication (cystic artery pseudoaneurysm) and $3.5 \%$ readmission in the second phase which was also due to wound related problems and surgical complication (CBD injury). ${ }^{10}$ These findings are consistent with our results showing that the main causes for the patients being nondischargeable even at 24 hours are difficult surgeries and surgical complications.

Of the total 88 patients, 60 patients were discharged at 24 hours, 10 patients were not dischargeable, and 18 patients were not discharged due to various factors such as social factors, logistic factors, presence of drain, patient preference, surgeon anticipated complications, and surgical complications. This is similar to the results of several other previous published studies. ${ }^{9-18}$ Although several ${ }^{9,10,12,13,17-20}$ had shown postoperative emesis as one of the major causes for nondischargeability, none of our patients had postoperative nausea and vomiting leading to nondischargeability. Eighteen patients who were dischargeable were not discharged because of various factors such as continuation of IV antibiotics (1), logistic insurance (4), patient factor due to patients unwillingness for discharge (1), presence of drain (7), social factors such as patient living at a far distance (1), surgeon anticipating complication (1), and surgical complication (1).

The main drawback of this study is that although we have seen for the dischargeability of the patients at 6 hours, we have not really discharged the patients. In our study, none of the patients who were dischargeable at 6 hours developed any complications during their stay at the hospital which would mean if at all these patients were discharged, these patients might not have required readmission. But this might be attributed to the good inpatient care provided at the hospital, and if these patients were discharged at 6 hours in real situation, they might have required readmissions.

\section{ConCLUSION}

Of the total 88 patients analyzed, 57 (64.7\%) patients were dischargeable at 6 hours. We can conclude that $65 \%$ of all the laparoscopic cholecystectomies can be performed as DCLCs if stringent selection criteria are followed. From our study, we can conclude that it will be safe to go for day care laparoscopic cholecystectomies in majority of patients except those with acute cholecystitis. Patients requiring an operative time more than 104 minutes should be observed for 24 hours.

$88.6 \%$ patients were dischargeable at 24 hours. Surgeon should anticipate a delayed discharge in patients who had a difficult surgery or when drain was used. All the patients who underwent laparoscopic cholecystectomies without these two factors were dischargeable by 24 hours. Even in patients where drain is used should be considered for early discharge if the PADSS criteria are met and the drain can be removed at a later date on OPD basis.
In our study, 13 patients of the 59 patients who were dischargeable at 24 hours were not discharged because of various factors such as continuation of IV antibiotics (1), logistic factor (insurance) (4), patient factor (1), presence of drain (5), social factor such as patient living at a far distance (1), and as surgeon anticipated complications (1).

Appropriate antibiotic usage, surgeon and patient awareness about the treatment protocols, and streamlining hospital processes can mitigate some of these factors delaying discharge. This will have a significant impact in cutting off the hospital costs as well as in preventing hospital-acquired infections.

\section{ACKnOWLedgments}

We are thankful to our statistician Mr Souvik Dutta who helped us immensely throughout the analysis for this study.

\section{References}

1. Keus F, de Jong J, Gooszen HG, et al. Laparoscopic versus open cholecystectomy for patients with symptomatic cholecystolithiasis. Cochrane Database Syst Rev 2006;(4):CD006231. DOI: 10.1002/ 14651858.CD006231.

2. Kiviluoto T, Siren J, Luukkonen P, et al. Randomised trial of laparoscopic versus open cholecystectomy for acute and gangrenous cholecystitis. The Lancet. 1998;351(9099):321-325.

3. Nicoll JH. The surgery of infancy-I. Pediatr Anesth 1998;8(3):248. DOI: 10.1046/j.1460-9592.1998.00534.x.

4. Marshall SI, Chung F. Discharge criteria and complications after ambulatory surgery. Anesth Analg 1999;88(3):508-517. DOI: 10.1213/00000539-199903000-00008.

5. LledóJB, Planells M, Espí A, etal.Predictive model offailure of outpatient laparoscopic cholecystectomy. Surg Laparosc Endosc Percutan Tech 2008;18(3):248-253. DOI: 10.1097/SLE.0b013e31816de922.

6. Psaila J, Agrawal S, Fountain U, et al. Day-surgery laparoscopic cholecystectomy: factors influencing same-day discharge. World J Surg 2008;32(1):76-81. DOI: 10.1007/s00268-007-9225-x.

7. Rayo A, Polanco A, Qiu S, et al. Safety of outpatient laparoscopic cholecystectomy in the elderly: analysis of 15,248 patients using the NSQIP database. J Am Coll Surg 2013;217(6):1038-1043. DOI: 10.1016/j. jamcollsurg.2013.08.001.

8. Chauhan A, Mehrotra M, Bhatia PK, et al. Day care laparoscopic cholecystectomy: a feasibility study in a public health service hospital in a developing country. World J Surg 2006;30(9):1690-1695. DOI: 10.1007/s00268-006-0023-7.

9. Bona S, Monzani R, Romario UF, et al. Outpatient laparoscopic cholecystectomy: a prospective study on 250 patients. Gastroenterol Clin Biol 2007;31(11):1010-1015. DOI: 10.1016/S0399-8320(07)78322-7.

10. Sherigar JM, Irwin GW, Rathore MA, et al. Ambulatory laparoscopic cholecystectomy. JSLS 2006;10(4):473.

11. Chang SK, Tan WB. Feasibility and safety of day surgery laparoscopic cholecystectomy in a university hospital using a standard clinical pathway. Singapore Med J 2008;49(5):397-399.

12. Victorzon M, Tolonen $\mathrm{P}$, Vuorialho T. Day-case laparoscopic cholecystectomy: treatment of choice for selected patients? Surg Endos 2007;21(1):70-73. DOI: 10.1007/s00464-005-0787-0.

13. Barthelsson C, Anderberg B, Ramel S, et al. Outpatient vs inpatient laparoscopic cholecystectomy: a prospective randomized study of symptom occurrence, symptom distress and general state of health during the first post-operative week. J Eval Clin Pract 2008;14(4):577584. DOI: 10.1111/j.1365-2753.2007.00920.x.

14. Hosseini SN, Mousavinasab SN, Rahmanpour H. Evaluate the outcome and identify predictive failure of outpatient laparoscopic cholecystectomy. J Pak Med Assoc 2009;59(7):452.

15. Marinis A, Stamatakis E, Tsaroucha A, et al. Safety and effectiveness of outpatient laparoscopic cholecystectomy in a teaching hospital: 
a prospective study of 110 consecutive patients. BMC Res Notes 2010;3(1):207. DOI: 10.1186/1756-0500-3-207.

16. Tuvayanon W, Toskulkao T, Asdornwised U, et al. Factors impacting readiness to discharge time from recovery room after laparoscopic cholecystectomy. Thai Surg 2011;32:53-59.

17. Sato A, Terashita Y, Mori Y, et al. Ambulatory laparoscopic cholecystectomy: an audit of day case vs overnight surgery at a community hospital in Japan. World J Gastrointest Surg 2012;4(12):296. DOI: 10.4240/wjgs.v4.i12.296.
18. Gelmini R, Franzoni C, Saviano M. Day surgery laparoscopic cholecystectomy: initial experience in 43 consecutive patients. Ann Ital Chir 2013;84(6):631-636.

19. Al-Qahtani HH, Alam MK, Asalamah S, et al. Day-case laparoscopic cholecystectomy. Saudi Med J 2015;36(1):46. DOI: 10.15537/ smj.2015.1.9738.

20. Sözen S, Özdemir CŞ. Day-case laparoscopic cholecystectomy: is it a safe and feasible procedure? Eur J Gen Med 2010;7(4):372-376. DOI: 10.29333/ejgm/82888. 